\title{
Product Maintainability Design Method and Support Tool Based on Feature Model
}

\section{Yufeng DING}

School of Mechanical and Electrical Engineering, Wuhan University of Technology, Wuhan, China. Email: dingyf@whut.edu.cn

Received June $5^{\text {th }}$, 2009; revised June 29 $9^{\text {th }}$ 2009; accepted July $7^{\text {th }}, 2009$.

\begin{abstract}
Maintainability is an important character which is given by product design process. The maintainability design criteria and measure index used in product maintainability analysis are summarized and discussed in this paper. A product maintainability design method is studied by integrating the product feature model, maintainability design criteria with measure index. Product feature model can be built on the basis of the product feature library quickly. Product feature library for steam turbine design is created by using SolidWorks design library origination structure. A methodology which supports the design and development of product maintainability design support tool (PMDSTs) is put forward. The function of PMDSTs is designed by using UML (Unified Modeling Language) use case diagram, it is developed by using $V C++6.0$. The maintainability analysis application case of steam turbine-generator system is given at last.
\end{abstract}

Keywords: Maintainability Design, Product Design Process, Steam Turbine, Solidworks

\section{Introduction}

If a product has poor maintainability, the maintenance activities which have to be performed on it during its life cycle are difficult, it will result in increasing the costs, and also more time is required to accomplish the maintenance tasks. Designs for maintainability had played an important role in the complex product deign process. Maintainability is the probability that required maintenance will be successfully completed in a given time period. It is a design characteristic that affects accuracy, ease, and time requirements of maintenance actions. It may be measured by combining factors such as frequency of maintenance, maintenance costs, elapsed maintenance or repair times, and labor hours etc.

Design for maintainability requires a product that is serviceable and supportable - better yet if the design includes a durability feature called reliability (absence of failures) then you can have the best of all worlds [1].

Maintainability is an important character which is given by product design, it makes easy to be repaired for the mechanical system. It has a specific effect on the maintenance cost of mechanical systems [2].

In order to realize product design for maintainability, some approaches and software tools have been studied and developed. A maintainability evaluation approach based on fuzzy logic is presented in [3], fuzzy linguistic variables are employed in order to represent and handle the design data available early in the design process. The measure tool of the product maintainability is developed. Maintainability and safety indicators at design stage for mechanical products are studied in [4]. The assessment procedure uses product 3D (Dimension) CAD (Computer Aided Design) model and associated semantic matrix gathering information from the product components' criticality and reliability.

\section{Product Integration Maintenance Model}

Product CAD model doesn't include product maintenance feature information; it can not provide support for product maintenance. So it needs to build product integration model by combining CAD model and maintenance feature information. Product integration model is composed of shape feature $F_{s}$, heat treatment feature $F_{h}$, management feature $F_{m}$ and maintenance feature $F_{m a}$. They can be united altogether to build a whole product feature model. The product feature classification is shown in the Figure 1. Maintenance feature is used for describing maintainability design and relevant information. It includes maintenance organization feature, maintainability feature, maintenance resource, maintenance process, fault feature, using demand, maintenance program, maintainability qualitative demand, quantitative demand, maintainability design analysis result, person model info etc. Maintainability program includes objectives, organization, maintainability design criteria, poli- 
cies and procedures, organizational interfaces, program tasks, evaluation and subcontractor/supplier activity etc. [5].

Product maintenance integration model is shown in Figure 2. $\mathrm{C}_{\mathrm{i}}$ means ith component. It can be a part or a sib-assembly. $\mathrm{R}(\mathrm{i}, \mathrm{j})$ represents the assembly relation between ith component and jth component. It is marked in the link arc between ith component and jth component.
Every component has an attribute table, all attribute tables are saved in the attribute table. Every attribute table file saves all feature informations of component $\mathrm{C}_{\mathrm{i}}$. Some data files such as design parameter table (excel file), design intent (word file), maintenance plan (Microsoft project file) are stored in design document data or maintenance data. They have a link in the Tag file. Tag file will look for the relevant file by the link [5].

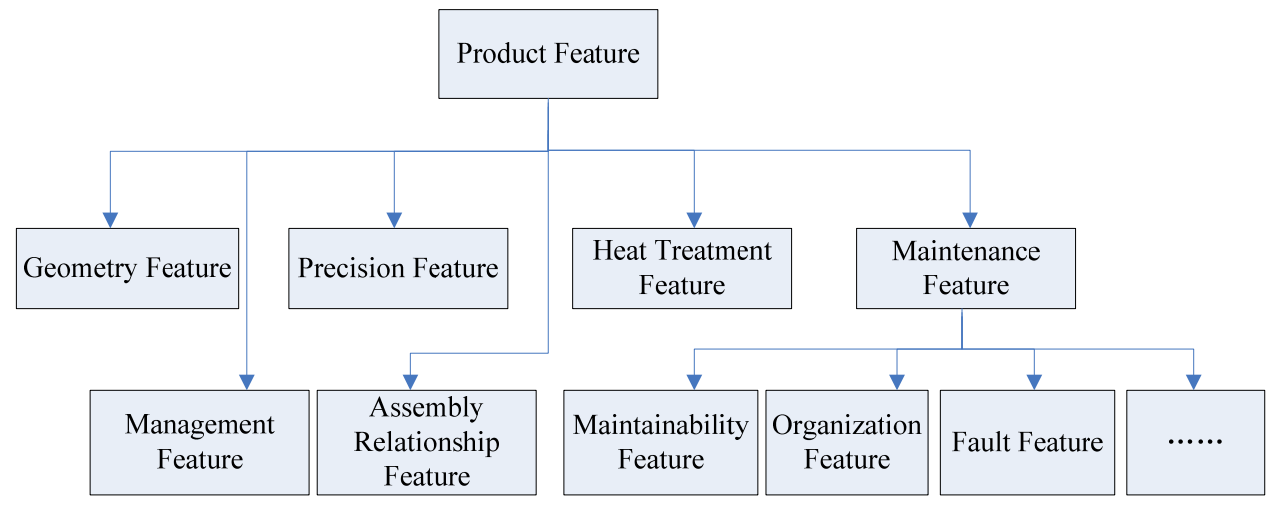

Figure 1. Product feature classification

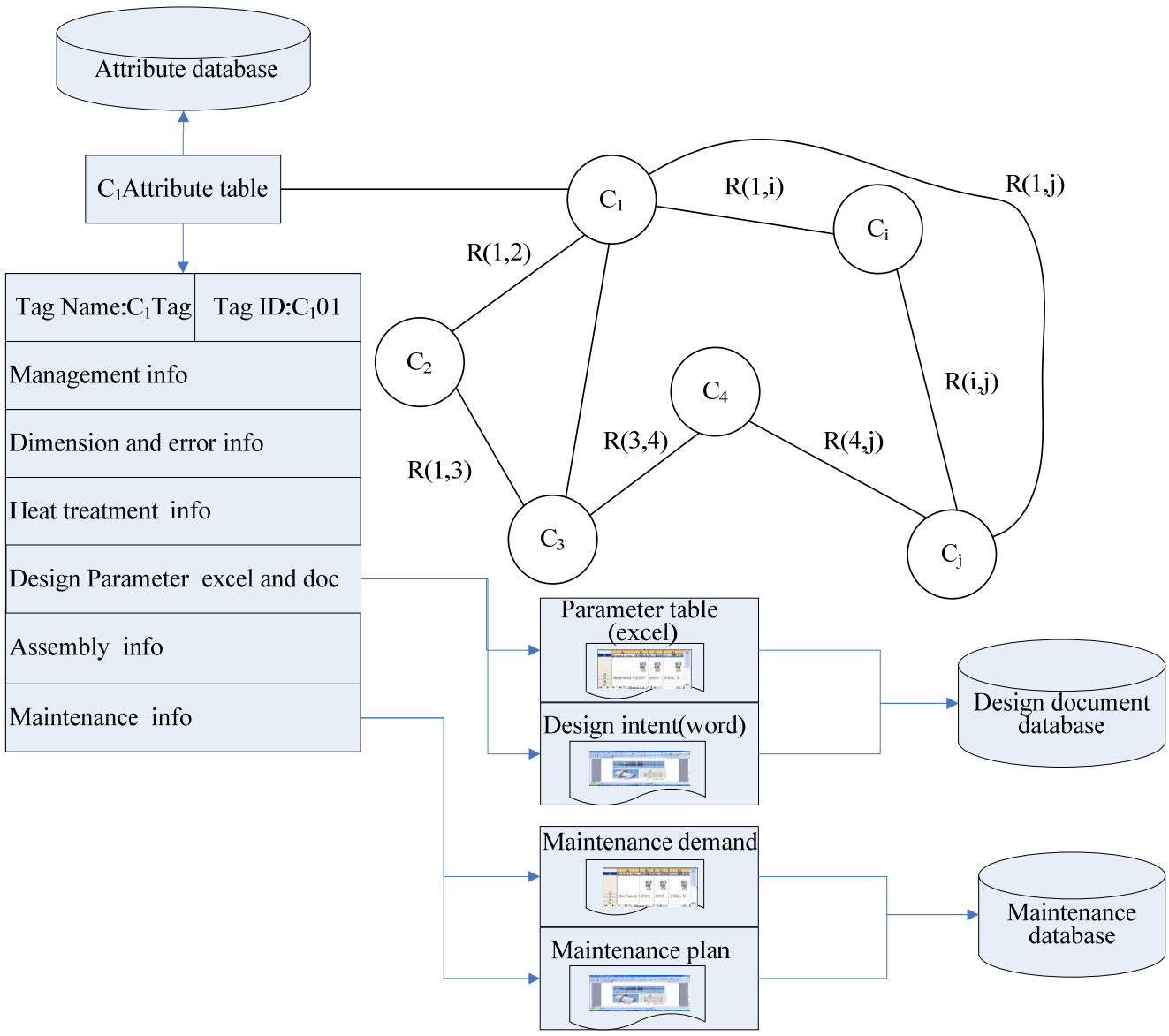

Figure 2. Product maintenance integration model 


\section{Design for Maintainability}

\subsection{The Principles of Design for Maintainability}

The design engineer is anxious to verify if product maintenance tasks can be accomplished with the available maintenance logistics and evaluate the performance of these tasks in design stage. The designer must change his/her design solution if the maintenance tasks are difficult, or even impossible to be accomplished in the given conditions. Some of the important general design guidelines that maintainability professionals have developed are shown in Figure 3 [6].

Maintainability criteria are composed of intrinsic criteria and contextual criteria [4]. Intrinsic criteria includes repairability, ability to be repaired after failure or damage; accessibility, easiness to reach a component inside the assembly; assemblability, ability to be assembled from an assembly; disassemblability, ability to be removed from an assembly; standardization, standard component or equipment; interchangeability, ability to be replaced with another component; survivability, ability of the product to continue to work after the failure of a considered component. Contextual criteria includes redundancy, for components existing in multiple equivalent occurrences; Competencies, human required to diagnose and to repair; Toolings, maintenance equipments like keys, screwdrivers...; Logistics, delivery of spare parts, transportation of maintenance team...; Environment, working conditions like lighting, temperature...; Delectability, easiness to detect failure and components concerned with; Testability, ability for a component or a sub-system to be tested...; Maneuverability, ability for a component or sub-system to be handled; Auto diagnostic: ability for a system to perform self-testing procedures.

\subsection{Maintainability Measure}

Maintainability, as a characteristic of design, can be de- fined on the basis of a combination of the following factors. They are Maintenance times, Maintenance frequency and Maintenance cost. The three factors are dependant on the fact that the system is operated and maintained in accordance with prescribed procedures and resources. From a systematic perspective, maintenance includes corrective maintenance and preventive maintenance. And from a software perspective, maintenance includes adaptive maintenance and perfective maintenance.

There are several approaches to evaluate the maintainability of a product at the design stage. They are maintainability design checklists, maintainability evaluation using physical mock-ups, maintainability evaluation using digital mock-ups and virtual reality and maintainability evaluation using quantitative approaches.

The measures used in maintainability analysis include mean time to repair, mean active preventive maintenance time, and mean active corrective maintenance time, maximum corrective maintenance time, and mean maintenance downtime [7].

\subsubsection{Mean Time to Repair (MTTR)}

Maintenance time to repair (MTTR) is very crucial and it depends mainly on the product configurations. MTTR measures the elapsed time required to perform a given maintenance activity and is subsequently used to calculate system availability and downtime. Exponential, lognormal, and normal probability distributions can all represent mean time to repair. The normal distribution is normally assumed for mechanical or electromechanical equipment with a remove-and-replace maintenance concept.

$$
T_{m t r}=\left(\sum_{i}^{m} \lambda_{i} T_{i}\right) / \sum_{i}^{m} \lambda_{i}
$$

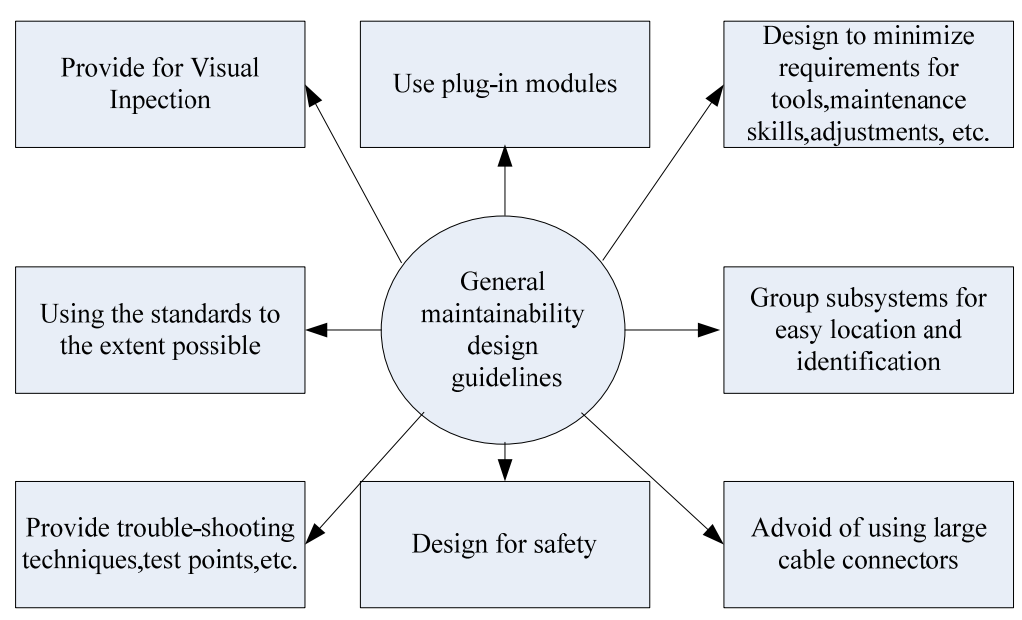

Figure 3. General maintainability design guidelines 


\subsubsection{Mean Preventive Maintenance Time}

Preventive maintenance activities such as inspections, calibrations, and tuning keep equipment at a specified performance level. The objective of a preventive maintenance program is to postpone the point at which the equipment or any of its components wears out or breaks down.

$$
T_{m p}=\frac{\sum_{i}^{k}\left(T_{m p i}\right)\left(F_{p t i}\right)}{\sum_{i}^{k} F_{p t i}}
$$

$\mathrm{T}_{\mathrm{mp}}$ is the mean preventive time. $\mathrm{T}_{\mathrm{mpi}}$ is the elapsed time for preventive maintenance task $\mathrm{i}$, for $\mathrm{i}=1,2,3 \ldots \ldots \mathrm{k} \cdot \mathrm{F}_{\mathrm{pti}}$ is the frequency of preventive maintenance task $i$, for $i$ $=1,2,3, \ldots, \mathrm{k} . \mathrm{k}$ is the number of preventive maintenance tasks.

\subsubsection{Median Corrective Maintenance Time}

Mean corrective time is a composite value representing the arithmetic average of the individual maintenance cycle times. Maintainability is the ability of a product to be maintained. Calculation of the median corrective maintenance time depends on the distribution describing time to repair. The median corrective maintenance time for lognormal distributed repair time is given by

$$
T_{\text {med }}=T_{\text {mtr }} / \exp \left(\sigma^{2}\right)
$$

$\sigma^{2}$ is the variance around the mean value of the natural logarithm of repair time.

\subsubsection{Maximum Corrective Maintenance Time}

This measures the time required to complete all potential repair activities up to a given percentage, often the 90th or 95th percentiles. The maximum corrective maintenance time for lognormal distributed is

$$
T_{m c m}=\operatorname{antilog}\left(T_{m}+k \sigma\right)
$$

$\mathrm{T}_{\mathrm{mcm}}$ is the maximum corrective maintenance time. $\mathrm{Tm}$ is the mean of the logarithms of the repair times. $\sigma$ is the standard deviation of the logarithms of repair times. $\mathrm{k}$ is equal to 1.28 or 1.65 for the 90th and 95th percentiles.

\subsubsection{Mean Maintenance Downtime}

This is the total time needed either to restore equipment to a specified performance level or to maintain it at that level of performance. Thus it includes active corrective and preventive maintenance times, administrative and logistic delay times.

$$
T_{\text {mmd }}=T_{\text {mam }}+T_{a d}+T_{l d}
$$

$\mathrm{T}_{\mathrm{mmd}}$ is the mean maintenance downtime. $\mathrm{T}_{\mathrm{mam}}$ is the mean active maintenance time, or mean time required to conduct corrective and preventive maintenance related tasks. $T_{\text {ad }}$ is the administrative delay time. $T_{\text {ld }}$ is the lo- gistic delay time.

\subsubsection{Maintenance Function}

The maintainability functions are used to predict the probability that a repair, beginning at time $\mathrm{t}=0$, will be accomplished in a time t. The maintainability function, $\mathrm{m}(\mathrm{t})$ for any distribution is expressed by

$$
m_{t}=\int_{0}^{t} f_{r}(t) d t
$$

$\mathrm{t}$ is time. $\mathrm{f}_{\mathrm{r}}(\mathrm{t})$ is the probability density function of the repair time.

\subsubsection{Maintenance Accessibility Evaluation}

Accessibility is the relative ease with which a part or piece of equipment can be reached for service, replacement, or repair. The lack of accessibility is an important maintainability problem and a frequent cause of ineffective maintenance.

The evaluation may also be performed by assigning to each maintainability criterion, a numerical value between 0 and 1 using a table, like the one listed in Table 1 [3].

\subsection{Maintainability Cost Analysis}

Maintainability is an important factor in the total cost of equipment. An increase in maintainability can lead to reduction in operation and support costs. For example, a more maintainable product lowers maintenance time and operating costs. Furthermore, more efficient maintenance means a faster return to operation or service, decreasing downtime.

The data to be input into a life cycle cost model include the purchase price of the product, mean time between failures (MTBF), MTTR, average material cost of a failure, labor cost per preventive maintenance action, labor cost per corrective maintenance action, installation costs, training costs, the warranty coverage period cost of carrying spares in inventory, and shipment forecasts over the course of the product's useful life [8].Corrective Maintenance cost estimation model estimates the corrective maintenance labor cost for a piece of equipment. The annual cost is expressed by

Table 1. Accessibility evaluation

\begin{tabular}{l|c}
\hline \multicolumn{1}{c|}{ Accessibility } & Value \\
\hline $\begin{array}{l}\text { All the parts are directly accessible and placed in the same } \\
\text { area }\end{array}$ & 1 \\
\hline $\begin{array}{l}\text { All the parts are directly accessible and placed in different ar- } \\
\text { eas }\end{array}$ & 0.8 \\
\hline $\begin{array}{l}\text { Some parts are not directly accessible, but those parts are } \\
\text { maintenance free }\end{array}$ & 0.6 \\
\hline $\begin{array}{l}\text { Some parts are accessible after disassembling a fast disassem- } \\
\text { bling entity (a screw, etc.) }\end{array}$ & 0.4 \\
\hline $\begin{array}{l}\text { The majority of the parts is accessible by disassembling one or } \\
\text { more entities }\end{array}$ & 0 \\
\hline
\end{tabular}




$$
C_{C M}=\frac{(\mathrm{SOH})\left(\mathrm{C}_{\mathrm{L}}\right)\left(\mathrm{T}_{\mathrm{mttr}}\right)}{\mathrm{T}_{\mathrm{mtbf}}}
$$

SOH represents the scheduled operating hours of the equipment. $\mathrm{C}_{\mathrm{L}}$ is the maintenance labor cost per hour. $\mathrm{T}_{\mathrm{mtbf}}$ is the mean time between failures for the equipment. $\mathrm{T}_{\mathrm{mttr}}$ is the mean time to repair for the equipment.

\subsection{System Reliability Analysis}

Reliability can be defined as the probability that a system will perform properly for a specified period of time under a given set of operating conditions. Implied in this definition is a clear-cut criterion for failure.

Let $R_{i}$ be the reliability of subsystem $i$ and $r_{i j}$ be the reliability of component, in subsystem $\mathrm{j}$,

$$
\begin{gathered}
1 \leq j \leq n_{i} \mathrm{i}=1,2, \ldots, \mathrm{k} \text {. Then } \\
R_{i}(t)=\prod_{j=1}^{n_{i}} r_{i j}(t)
\end{gathered}
$$

The system reliability, say $\mathrm{R}(\mathrm{t})$, is given by

$$
R_{i}(t)=1-\prod_{i=1}^{k}\left(1-\prod_{j=1}^{n_{i}} r_{i j}(t)\right)
$$

The system consists of $\mathrm{k}$ subsystems connected in parallel, with subsystem $i$ consisting of $n_{i}$ components in series for $i=1,2, y, k$. Such a system is called a series-parallel system [9]. Figure 4 shows the diagram of a series-parallel system [10].

The system mean time to failure (MTTF) can be derived in the following form:

$$
T_{m t t f}=\frac{1}{\lambda} \sum_{l=1}^{k} \sum_{1 \leq i_{1}<\ldots \leq k} \frac{(-1)^{l+1}}{n_{i}^{(l)}}
$$

\section{The Development and Application of Product Maintainability Design Support Software}

\subsection{Product Feature Library}

A library feature is a frequently used feature, or combination of features. Many features can be created once and then save in a library for future use. Product feature is organized according to product feature classification of Figure 1.The product feature can be built and saved in

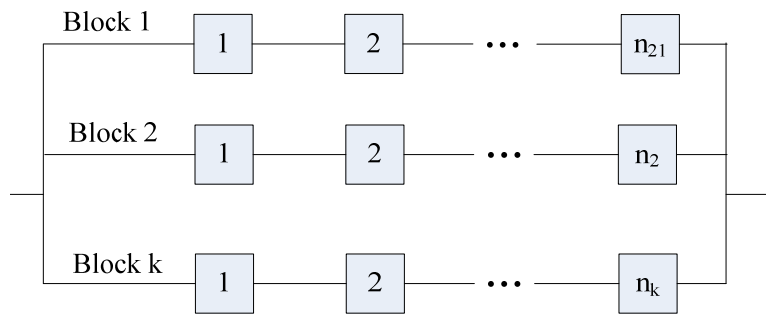

Figure 4. Series-parallel system design library of Solidworks. The directory structure can be built in the directory of install_directoryldataldesign library. To create a library feature that includes references, it is needed to dimension the library feature relative to the base part on which designer create it. References create dimensions used to position the library feature $\left({ }^{*}\right.$.sldlfp) on the model $\left({ }^{*}\right.$.sldprt). Steam turbine feature library in the SolidWorks is shown in Figure 5.

\subsection{Product Feature Modelling Based on Feature Library}

Industry steam turbine is composed of about tens of thousands of parts. The rotor, blade and cylinder of steam turbine work under the condition of high temperature and impulsion. They have high manufacture precision. The rotor of steam turbine is the most important, highest precision and most complicated part in the steam turbine product. Rotor includes thousands of dimensions.

For example, steam turbine rotor axis can be divided into five parts, they are front axis feature $\left(\mathrm{A}_{\mathrm{FA}}\right)$, front seal feature $\left(A_{F G S}\right)$, whole blade wheel feature $\left(A_{B W}\right)$, back seal feature $\left(A_{B G S}\right)$ and back axis feature $\left(A_{B A}\right)$.It can be represented in equation (11).

$$
\mathrm{Fr}=\mathrm{A}_{\mathrm{FA}} \cup A_{\mathrm{FGA}} \cup A_{C A} \cup A_{B G A} \cup A_{B A}
$$

All features of steam turbine rotor can be organized in the design feature library in the SolidWorks. But the first step must be done is to classify the features according to component classification and recognize the feature dimension. Some dimension relation can be built in some equation. The maintenance feature information had been organized into attribute table which is integrated with the geometry feature. Steam turbine feature library in the SolidWorks is shown in Figure 5.

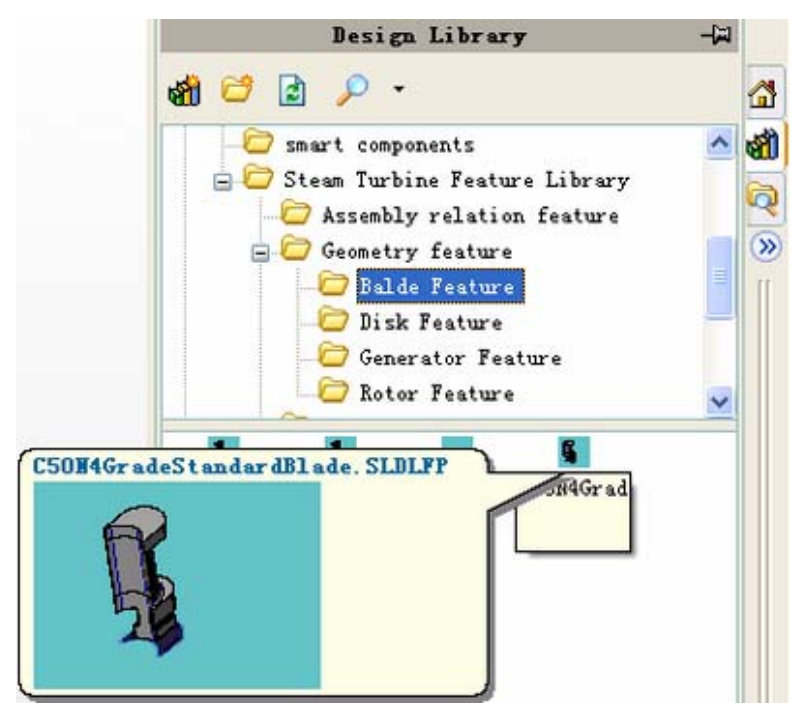

Figure 5. Steam turbine feature library in the SolidWorks 


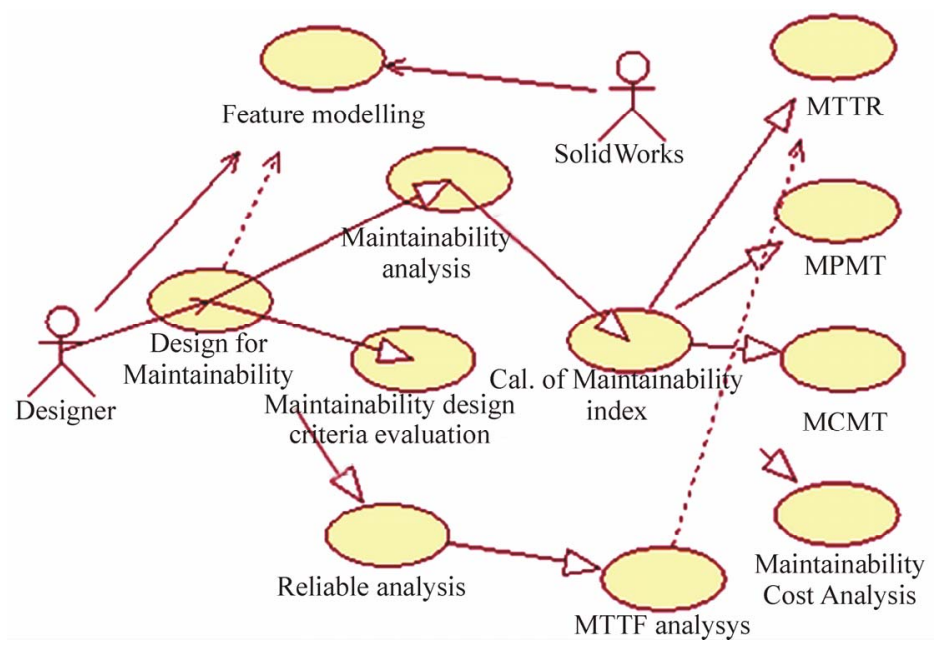

Figure 6. The use case diagram of product maintainability design support tool

\subsection{The Development Process of PMDSTs}

PMDSTs is a typical customization software. It can not be run without 3D CAD software SolidWorks platform. The development process of product maintainability design support software obeys following methodology.

1) Design the function of product maintainability, design support tool by using use case diagram.

2) Build main framework DLL (dynamic link library) using SolidWorks COM Add-In Wizard.

3) Build every function module DLL in C++ language.

4) Call every function module DLL in main DLL framework by using LoadLibrary method.

5) Test software function and performance by using SolidWorks Add-in interface.

The use case diagram of product maintainability design support tool is shown in Figure 6.The PMDSTs include feature modeling, design for maintainability and reliable analysis use case etc. Product feature model is built in the 3D CAD software Solidworks. Design for maintainability and reliable analysis is carried out based on the product feature modeling. The part feature information and assemble feature relation can be extracted by using Solidworks API (Application Program Interface) function.

The system is developed by using Visual C++ 6.0 and Microsoft SQL Server 2000. Product maintainability design criteria are stored in the Database. The database is connected using ODBC (Open database Connectivity). The maintainability design support software is a kind of C/S (Client/Server) software. Windows 2000 Server OS (Operation system) is installed in the server computer. Windows XP OS is installed in Client computer. The main framework DLL is created into a visual C++ DLL or visual $\mathrm{C}++$.NET DLL add-in using the SolidWorks COM Add-In Wizard included in the SolidWorks API SDK (Software Development Kit) [11].
The function StartApplication and TerminateApplication which is used to connect the solidWorks and terminate the PMDST are as follows.

bool CSteamTurbineSysApp::StartApplication(void)

$\{/ /$ add menus to the active document

AddMenus();

//Add toolbars

AddToolbars();

// create a control item to handle application-level events

swAppEvents* eventApp = new swAppEvents;

eventApp->OnCreate(m_pSldWorks); return TRUE;

\}

void CSteamTurbine-

SysApp::TerminateApplication(void)

$\{$ if (m_pSldWorks == NULL)

return;

// remove all menus

RemoveMenus();

//remove the toolbars

RemoveToolbars();

// release the PropertyManager object

ReleasePage();

LPMODELDOC pModDoc $=$ NULL;

HRESULT res $=$ TheApplica-

tion->GetSWApp()->get_IActiveDoc(\&pModDoc);

if $($ pModDoc $==$ NULL $)$

TheApplication->m_pActiveDoc = NULL;

int count $=\mathrm{m} \_$EventList.GetCount( $)$;

for (int $\mathrm{i}=0$; $\mathrm{i}<$ count; $\mathrm{i}++$ )

$\{$ CObject* headEvent $=$

m_EventList.GetHead();

delete headEvent;

if (m_pActiveDoc != NULL)

m_pActiveDoc->Release(); 


$$
\begin{aligned}
& \text { // disconnect from SolidWorks } \\
& \text { m_pSldWorks->Release(); } \\
& \text { m_pSldWorks = NULL; }
\end{aligned}
$$

Every function module DLL is called in main DLL framework by using ::LoadLibrary method is as follows.

void MaintainabilityAnalysis ()

\{// MaintainabilityAnalysis interface function

CString m_AppPath;

m_AppPath=GetAppPath();

CString strConnect;

LPSLDWORKS m_pSldWorks;

// strConnect="MTCADServer";

m_pSldWorks= TheApplication- $>$ GetSWApp();

LPMODELDOC2 pModDoc = NULL;

HRESULT res $=$

m_pSldWorks->get_IActiveDoc2(\&pModDoc);

LPPARTDOC pPartDoc $=$ NULL;

res $=$ pMod-

Doc->QueryInterface(IID_IPartDoc,(LPVOID

*)\&pPartDoc);

typedef void (WINAPI * SeriousDe-

gree)(LPMODELDOC2,LPPARTDOC,LPSLDWORKS)

;

HINSTANCE hmod;

hmod = ::LoadLibrary(_T("D:\\Steamturbine MaintenanceSoft \\MaintainabilityAnalysis.dll"));

if(hmod==NULL)

\{AfxMessageBox(_T("MaintainabilityAnalysis.dll

can not be found in current directory!")); \}

SeriousDegree lpproc;

lpproc = (SeriousDegree)GetProcAddress(hmod," MaintainabilityAnalysis ");

if(lpproc!=( MaintainabilityAnalysis)NULL)

(*lpproc)(pModDoc,pPartDoc,m_pSldWorks); \}

FreeLibrary(hmod);

\subsection{Maintainability Measure Index Analysis Case}

The process block diagram of a steam turbine-generator system is shown in Figure 7 [12]. In the design process of steam turbine-generator system, MTTR and MTBF etc. Maintainability measure index of steam turbine-generator system is calculated as follows.

$$
\begin{gathered}
T_{m t r}=\left(\sum_{i}^{m} \lambda_{i} T_{i}\right) / \sum_{i}^{m} \lambda_{i}=\frac{39227}{370.43}=105.9 \\
T_{m t b f}=\frac{10^{6}}{\sum \lambda}=2.699 \\
A=\frac{T_{m t b f}}{T_{m b b}+T_{m t r}}=96.2
\end{gathered}
$$

The calculation process of maintainability measure index of steam turbine-generator system is shown in Figure 8. Every Sub-system/assembly MTBF value must be

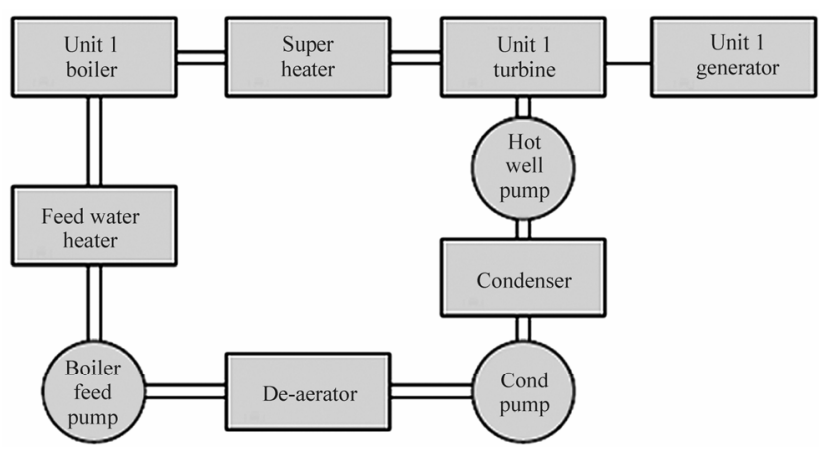

Figure 7. Process block diagram of a steam turbine-generator system

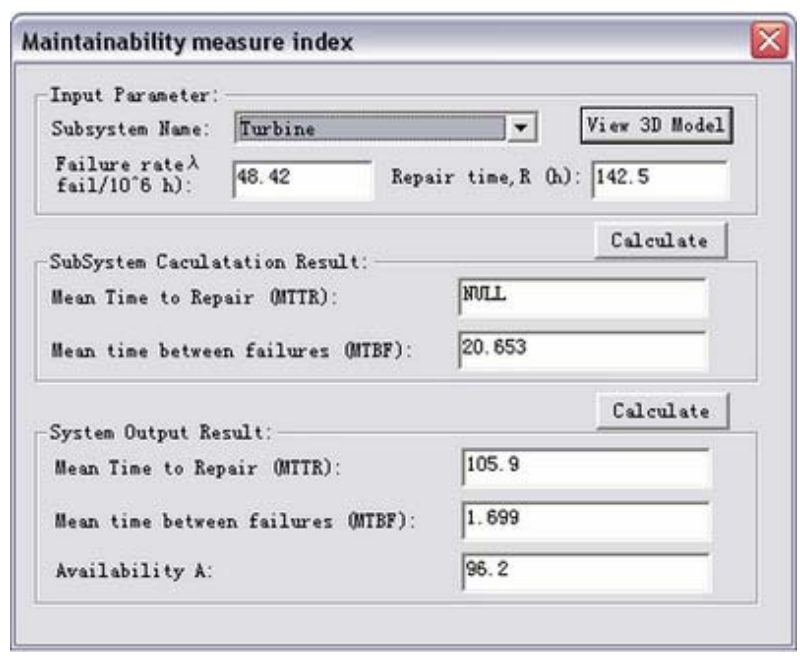

Figure 8. The calculation of maintainability measure index

calculated at first in order to get MTTR, MTBF and availability etc. index of steam turbine-generator system. The designer can read Solidworks 3D feature model of current subsystem by pressing 'View 3D Model' button.

\section{Conclusions}

In this paper, the maintainability design criteria and measure index used in product maintainability analysis are discussed. A product feature library for steam turbine design is built. The feature library can support steam turbine product feature modeling quickly. Product maintainability design method based on feature modeling can help to analyze product maintainability in the product design process. PMDSTs is developed by using Visual C++ 6.0 and Microsoft SQL Server 2000. PMDSTs can support designer to evaluate product maintainability by applying maintainability design criteria and maintainability measure index in the design stage. This method will help to enhance product maintainability efficiently. PMDSTs will be used to product computer support collaborative design (CSCD) process through the next step 
of developing new collaborative support function.

\section{Acknowledgment}

This paper is supported by the Key Technologies R\&D Program of Wuhan City (No. 200810321153) and Wuhan Youth Science and Technology Chenguang Program (No.200750731289).

\section{REFERENCES}

[1] http://www.barringer1.com/jul01prb.htm.

[2] B. Abdullah, M. S. Yusoff, and Z. M. Ripin, "Integration of design for modularity and design for assembly to enhance product maintainability,” Proceddings of 1st International Conference 7th AUN/SEED-Net Fieldwise seminar on Manufacturing and Material processing, pp. 263-269, University Malaya, 2006.

[3] C. A. Slavila, C. Decreuse, and M. Ferney, "Fuzzy approach for maintainability evaluation in the design process," Concurrent Engineering, Vol. 13, No. 4, pp. 291-300, 2005.

[4] A. Coulibaly, R. Houssin and B. Mutel, "Maintainability and safety indicators at design stage for mechanical products,” Computers in Industry, Vol. 59, No. 5, pp 438-449, 2008.
[5] Y. F. Ding and B.Y. Sheng, "Study on product maintenance integration model," Submit to The IEEE International Conference on Industrial Engineering and Engineering Management (IEEM) 2009.

[6] M. Pecht, "Product reliability, maintainability, supportability handbook,” CRC Press, Boca Raton, Florida, pp. 191-192, 1995.

[7] B. S. Dhillon, “Engineering maintainability,” Eelservier, 2008.

[8] H. Reiche, "Life cycle cost in reliability and maintainability of electronic systems,” Computer Science Press, Potomac, Maryland, pp. 3-23.1980,

[9] W. Kue, V. R. Parsad, F. A. Tillman and C. Hwang, "Optimal reliability design: Fundamentals and applications," Cambridge University Press, 2001.

[10] A. M. Sarhan, "Reliability equivalence factors of a general series-parallel system," Reliability Engineering and System Safety, Vol. 94, No. 2, pp.229-236, 2009.

[11] SolidWorks Corporation. "Solidworks 2000 API help," 2006.

[12] Stapelberg and R. Frederick, "Handbook of reliability, availability, maintainability and safety in engineering design,” Springer, 2009. 